\title{
Recent Advances in Biomarker
}

\section{Discovery - from Serum to Imaging- based Biomarkers for a Complex Assessment of Heart Failure Patients}

\author{
Andreea-Luciana Chiotoroiu, Corneliu-Florin Buicu, Claudiu Neagu, Theodora Benedek
}

University of Medicine and Pharmacy, Tîrgu Mureș, Romania

\section{CORRESPONDENCE \\ Corneliu-Florin Buicu \\ Str. Gheorghe Marinescu nr. 38 \\ 540139 Tîrgu Mureș, Romania \\ Tel: +40 265215551 \\ E-mail: florin_buicu@yahoo.com}

\section{ARTICLE HISTORY}

Received: 5 September, 2016

Accepted: 19 September, 2016
Andreea-Luciana Chiotoroiu • Str. Gheorghe Marinescu nr. 38, 540139 Tîrgu Mures, Romania, Tel: +40265215551

Claudiu Neagu • Str. Gheorghe Marinescu nr. 38 , 540139 Tîrgu Mureș, Romania, Tel: +40 265215551

Theodora Benedek • Str. Gheorghe Marinescu nr. 38 540139 Tîrgu Mures, Romania, Tel: +40 265215551

\begin{abstract}
Over the last years, a vast majority of serum biomarkers and imaging techniques have been used alone or combined in the diagnosis, management and prognosis of numerous pathologies. This review provides a brief insight into the novelties from the last 6 years (2010-2016) regarding serum and imaging markers in heart failure (HF). New information about natriuretic peptides (NPS), soluble ST2 (Sst2), growth differentiation factor 15 (GDF-15), myeloperoxidase (MPO), C-reactive protein (CRP), procalcitonin (PCT), troponins (Tns), myoglobin (Mb), galectin-3 (Gal-3), micro ribonucleic acids (microRNAs) and long non-coding ribonucleic acids (IncRNAs), copectin and cardiac magnetic resonance (CMR) measurements were summarized in this review in order to guide the practitioner.
\end{abstract}

Keywords: heart failure, biomarkers, cardiac imaging

\section{INTRODUCTION}

Heart failure (HF) represents one of the most frequent cardiovascular diseases and various serum biomarkers and imaging techniques have been developed for a proper assessment of its evolution. This review provides a brief insight into the novelties from the last 6 years (2010-2016) regarding serum and imaging markers characterising different stages of heart failure.

\section{NATRIURETIC PEPTIDES}

According to the BACH trial (Biomarkers in Acute Heart Failure), run in 15 clinical sites, several new natriuretic peptides (NPs) such as midregion pro-atrial NP (MR-proANP) can be a biomarker as valuable as the brain natriuretic peptides (BNP) for diagnosing acute heart failure (AHF) in subjects presenting with dyspnea, and they may also have clinical utility in unclear cases, when elevated 
levels of BNP are difficultly interpreted in the clinical context. Mid-regional pro-adrenomedullin (MR-proADM) represents another biomarker with an accuracy of $73 \%$ for predicting 90-day survival in HF patients, higher than the accuracy reported for BNP, which has been demonstrated to be only $62 \% .^{1}$

In a prospective study addressing the function of $\mathrm{N}$ terminal pro-B-type natriuretic peptide (NT-proBNP) in guiding the treatment options for cases with impaired left ventricle (LV) systolic function, NT-proBNP-guided therapy was associated with lower event rates, improved living conditions, and superior LVEF (left ventricular ejection fraction) compared to standard therapies, independent on the age of the subjects $(\mathrm{p}=0.03) .^{2}$

The Atherosclerosis Risk in Communities (ARIC) Study, recruiting 12,230 patients with a body mass index (BMI) of $\geq 18.5 \mathrm{~kg} / \mathrm{m}^{2}$ and no history of HF at baseline, followed-up their evolution for a median of 20.6 years, and showed that NT-proBNP represents a valuable biomarker that offers significant prognostic information regarding the risk of developing $\mathrm{HF}^{3}$

\section{SOLUBLE ST 2}

In a study conducted on 107 subjects with EF $>50 \%$, evaluating echocardiography-derived imaging biomarkers, serum NT-proBNP and soluble ST2 (sST2) levels in patients with arterial hypertension and HF, the multivariate analysis showed that an $\mathrm{sST} 2$ value $>13.5 \mathrm{ng} / \mathrm{mL}$ was independently connected to a normal ejection fraction, concluding that in addition to NT-proBNP, sST2 could be useful for a more complex risk assessment in heart failure patients with diastolic impairment of the left ventricle. ${ }^{4}$

ST2 proved to be superior to Gal-3, as biomarkers characterizing myocardial fibrosis in CHF. In a head-to-head evaluation of 3 biomarkers (sST2, growth differentiation factor (GDF)-15, and highly-sensitive troponin T - hsTnT) in 151 patients with CHF, the only biomarker that showed predictive power in foreseeing changes in the left ventricular function, was sST2. All these results indicate that sST2 is a useful biomarker in subjects with CHF.5,6

\section{GROWTH DIFFERENTIATION FACTOR 15}

In a study conducted on 269 subjects with coronary heart disease (CAD), out of which 98 had chronic HF, plasma growth differentiation factor 15 (GDF-15) showed higher levels in individuals with CAD and heart failure, as compared to those with CAD and no HF. Simultaneously, GDF-15 was positively correlated to NT-proBNP levels, and negatively correlated to the ejection fraction. Importantly, GDF-15 showed no association with gender, age, BMI, alcohol consumption, smoking, diabetes, or hypertension. ${ }^{7}$

Another study showed that sST2, galectin-3 and GDF15 were not correlated with other noncardiac diseases in patients with HF (such as pneumonia, chronic obstructive pulmonary disease [COPD], renal disease, sepsis). However, GDF-15 levels were strongly correlated with galectin-3. ${ }^{8}$

In a study that included 916 consecutive patients, the prognostic role of plasma GDF-15 vs. NT-proBNP in HF with preserved EF vs. reduced EF was evaluated. From the study population, 488 were retested after 6 months. The results showed similar levels of elevated GDF-15 regardless of the LVEF, while NT-proBNP was more elevated in subjects with HF. At the same time, the prognostic role of a $>20 \%$ increase in GDF-15 was a stronger predictor compared with a similar increase in the NT-proBNP levels. ${ }^{9}$

\section{MYELOPEROXIDASE}

In the Ludwigshafen Risk and Cardiovascular Health study, plasma levels of myeloperoxidase (MPO) and of eight MPO polymorphisms were measured in 3,036 subjects, and their evolution was followed-up for 7.75 years. The results of this study indicated that MPO does not hold any role in the progression of cardiovascular conditions but, it indicated a good correlation between MPO concentrations, and cardiovascular and all-cause death rates. ${ }^{10}$

In another prospective study, a significant association was demonstrated between MPO levels, heart-type fatty acid-binding protein (H-FABP) and troponin $\mathrm{T}(\mathrm{TnT})$ levels as markers of myocardial damage in HF patients. The results showed that MPO could have a say in the development and progression of myocardial injury in cases with CHF, even if MPO levels were not associated with TnT levels. ${ }^{11}$

\section{C-REACTIVE PROTEIN}

The role of C-reactive protein (CRP) in acute coronary syndromes, added to the GRACE risk score, was studied in a large study performed on 1,501 patients. In this study, an elevated CRP level was a moderate, but independent factor in predicting the 30-day mortality in acute coronary syndromes, even after correction for associated diseases, hemodynamic conditions, and therapeutical management, demonstrating that a joint approach based on addition of CRP test to the GRACE risk score can improve the accu- 
racy of risk assessment in subjects with heart failure and ACSs. ${ }^{12}$

A very large trial including 10,160 participants aimed to find the correlation between high-sensitivity CRP (hsCRP) and the risks of diabetes, cardiovascular disease or mortality at 6 years of follow-up. In this study, a significant increase or sustained elevation of serum hs-CRP during the 6-years, was directly linked to a higher risk for diabetes, ischemic heart disease, ischemic stroke, HF, and death. ${ }^{13}$

CRP was also linked to a higher possibility of thromboembolic events and mortality secondary to vascular events in atrial fibrillation (AF) patients. ${ }^{14}$

It has been demonstrated that for individuals with CAD and chronic HF with systolic dysfunction, the degree of the systemic inflammatory status quantified through serum CRP levels is directly correlated with the cardiopulmonary exercise performance. These results indicate the need for initiation of anti-inflammatory treatments in HF. ${ }^{15}$ Also, an older study from 2010 showed that hs-CRP can offer additional prognostic value for the risk stratification of CHF. 16

Another study of 966 patients with CHF showed that severe central sleep apnea (CSA) was associated with high levels of CRP, explaining thus the negative prognostic effect of sleep apnea. ${ }^{17}$

\section{PROCALCITONIN}

Procalcitonin (PCT) represents an inflammatory marker characteristic for early atherosclerosis. A study on 77 patients with ACS showed that an increased PCT in the first $48 \mathrm{~h}$ from admission in the hospital may be correlated to higher early and 6-month death rates. ${ }^{18}$ In acute myocardial infarction (AMI) survivors, PCT was also related with the rate of major adverse cardiac events (MACE), LV dysfunction and the remodeling process. ${ }^{19}$ In another study on 2,131 patients with CAD, PCT was related with a higher cardiovascular mortality, but without being superior to the CRP predictive value. ${ }^{20}$

A large prospective study on 3,713 patients without any previous history of cardiovascular disease, demonstrated a significant relationship between PCT levels and the rate of coronary events and cardiovascular mortality. At the same time, PCT was associated with other cardiovascular risk indicators such as CRP, arterial hypertension, diabetus mellitus, renal function, and showed to be inversely correlated with HDL levels and smoking. ${ }^{21}$

Regarding the role of PCT as a biomarker for heart failure patients, a retrospective smaller case-control study showed that serum PCT levels can differentiate between HF pa- tients and healthy controls with a sensitivity of $88.9 \%$ [95\% confidence interval 75.9-96.2\%] and a specificity of $100 \%$ [82.2-100.0\%]), thus demonstrating the role of PCT as a potential biomarker that can influence the evolution of HF.22

PCT has also proved to have an important value for the prediction of all-cause mortality or hospitalization at 3 months in individuals with acute decompensated HF (ADHF) without clinical indicators of infection upon admission..$^{23}$ As regards to patients with signs of bacterial infection at presentation, a study on 4,698 cases from China concluded that PCT expression should be carefully examined in elderly patients with an infection complicating $\mathrm{CHF}$, indicating a high positive predictive value of PCT decrease in assessing the evolution of HF. ${ }^{24}$

The importance of PCT values was also demonstrated by two other studies, one that emphasized the essential role of PCT in diagnosing pneumonia in the emergency department (ED) for patients with dyspnea, and another one that used a PCT-based algorithm in choosing the right treatment for patients with respiratory diseases, indicating that antibiotic treatment cannot replace diuretics and HF medication in symptomatic HF patients without a confirmed pulmonary infection..$^{25,26}$

\section{TROPONINS}

High-sensitive troponin $\mathrm{T}$ (hsTnT), a biomarker for myocardial necrosis, was evaluated in a prospective reasearch, conducted on 107 subjects with ADHF. In this study, hsTnT, NT-proBNP and sST2 offered complementary prognostic information in patients with ADHF, concluding that a panel containing these 3 biomarkers can provide superior risk stratification compared to the isolated use of each one. ${ }^{27}$

Another prospective study on 202 patients with ADHF demonstrated that hsTnT assay offered an overall comparable prognostic information to conventional troponin $\mathrm{T}(\mathrm{c} \operatorname{Tn} \mathrm{T})$. However, in unclear cases where c $\mathrm{Tn} T$ was less precise or negative, increased levels of hsTnT were able to provide the requested prognostic information. ${ }^{28}$

This has been also demonstrated in another study on 504 patients followed during a 5-year follow-up period, which showed higher detection rates in CHF, and independent and better prognostic value for mortality when using hsTnT assay as compared to standard assay. ${ }^{29}$

Regarding the isoforms of troponin, another prospective global multicenter study demonstrated that high-sensitivity cardiac troponin $\mathrm{T}$ (hs-cTnT) was more reliable for predicting long-term mortality than high-sensitive cardiac troponin I (hs-cTnI). ${ }^{30}$ 


\section{MYOGLOBIN}

Myoglobin ( $\mathrm{Mb}$ ) is a cardiac enzyme released by the injured myocardium. In its oxygenated state it protects the heart from excessive nitric oxide (NO) levels via scavenging NO, however, in hypoxic conditions it becomes deoxygenated $\mathrm{Mb}$, which has the opposite role, producing $\mathrm{NO}$ and protecting the myocardial cells from the lesions associated with myocardial ischemia/reperfusion injury. ${ }^{31}$

$\mathrm{Mb}$ is one of the first biomarkers released after an ACS, and an $\mathrm{Mb}$ concentration can be discovered in the peripheral blood immediately after an acute myocardial infarction. New point-of-care devices have been proposed for the detection of $\mathrm{Mb}$, used as a simple and reliable tool for the early diagnosis of AMI. ${ }^{32,33}$ Regarding the association between survival and serum concentrations of various biomarkers, a cohort study conducted on 310 patients with idiopathic dilated cardiomyopathy (IDC) showed that from a panel of 3 biomarkers (cardiac troponin I, creatine kinase, and $\mathrm{Mb}$ ) only cTnI proved to independently predict the all-cause death rates in IDC subjects. ${ }^{34}$

\section{GALECTIN-3}

Galectin-3 (Gal-3) is also a valuable biomarker in heart failure patients. In a small study that included 44 subjects with $\mathrm{HF}$ and 38 healthy controls, Gal-3 serum levels were correlated with other biomarkers characterizing ventricular function, such as serum NT-proBNP, hs-CRP levels and echocardiography-derived biomarkers. ${ }^{35}$

Gal-3 proved to have limited intraindividual biological variability as a biomarker in HF subjects. ${ }^{36}$

It was demonstrated that protein kinase C (PKC- $\alpha)$ increases Gal-3 levels, promoting cardiac fibrosis and $\mathrm{HF}$, and the effect of angiotensin II (Ang II) could be partly mediated by the activation of the PKC-Gal-3 pathway. ${ }^{37}$

Serum levels of Gal-3 have been shown to be increased in both CHD and HF patients, and to independently predict the all-cause death and re-hospitalization rates caused by new episodes of decompensated HF. Gal-3 levels also had a better prognostic role in HFpEF patients than in HFrEF patients. ${ }^{38}$

\section{MICRO RIBONUCLEIC ACIDS AND LONG NON-CODING RIBONUCLEIC ACIDS}

MicroRNA (miRNA) have been proposed to serve as circulating biomarkers in the early stages of CHF, and can also represent therapeutic targets in these patients. A recent study indicated that selected micro ribonucleic acids
(microRNA) involved in apoptosis, hypertrophy and fibrosis are up-regulated in the myocardium of patients with a history of HF episodes, in contrast to those without HF. ${ }^{39}$

A study on a small number of patients showed that a panel of miRNAs may have a prognostic role in predicting heart failure following an AMI, identifying patients at risk for $\mathrm{LV}$ remodeling. ${ }^{40}$

The role of mitochondrial long non-coding ribonucleic acid (mitocondrial IncRNA) uc022bqs.1 (LIPCAR) has been analysed in a prospective research, carried out in multiple centers, which demonstrated that LIPCAR was upregulated in patients with $\mathrm{CHF}$, and that higher levels of LIPCAR were linked to a higher cardiovascular mortality. ${ }^{41}$

\section{COPEPTIN}

Copeptin has been proposed as a new biomarker associated with HF, after a study on 145 patients undergoing successful primary percutaneous transluminal coronary angioplasty (PTCA), who exhibited a distinct release pattern for this biomarker, with a peak in the first hour after the debut of an acute coronary event. ${ }^{42}$

The association of hs-cTnT with copeptin in a common biomarker panel slightly increased the rate of ACS detection at admission. ${ }^{43}$

A more recent study of copeptin on 195 patients with a 5-year follow-up demonstrated that copectin is an independent long-term prognostic indicator in HfrEF patients. ${ }^{44}$

\section{RECENT IMAGING-BASED BIOMARKERS}

Right ventricular (RV) function is currently evaluated using standard and pulsed Doppler tissue cardiac ultrasonography and magnetic resonance imaging (MRI). Tricuspid annular plane systolic excursion, systolic longitudinal velocity, tissue strain, and 2-dimensional (2D) strain) proved to be valuable imaging biomarkers for the assessment of RV function, showing a good correlation with the MRI calculated RV EF (RVEF). ${ }^{45}$

LA emptying function (LAEF) was also identified as a relevant biomarker associated with left and right ventricular function, patients with low LAEF exhibiting lower LV and RV EF, greater LV and RV mass, and increased serum NTproBNP levels. This biomarker has been proved to represent a powerful predictor of adverse outcomes, independent of other biomarkers characterizing cardiac dysfunction. ${ }^{46}$

The diagnostic value of cardiac MRI (CMR) with late gadolinium enhancement (LGE) for the evaluation for HF with ischemic versus nonischemic etiology has also been 
studied in several trials. An ischemic pattern on both LGE and cine sequences diagnosed ischemic cardiomyopathy with a specificity of $87 \%$, showing a great potential in differentiating the ischemic form of LV cardiomyopathy from its nonischemic form. ${ }^{47}$

Several studies suggested new imaging-based biomarkers to assess the ventricular functions, such as late heartto-mediastinum ratio (HMR) during cardiac metaiodobenzylguanidine (MIBG) imaging in the ADRECARD study, or (123)I-metaiodobenzylguanidine ((123)I-MIBG) imaging in the ADMIRE-HF study (AdreView Myocardial Imaging for Risk Evaluation in Heart Failure). ${ }^{48,49}$

The potential role of CMR-derived T1 mapping in evaluating myocardial fibrosis was demonstrated by several studies, which showed that this new biomarker can independently predict invasively measured LV stiffness. ${ }^{50}$

\section{CONCLUSION}

Serum biomarkers such as natriuretic peptides, soluble ST2, growth differentiation factor 15, myeloperoxidase, C-reactive protein, procalcitonin, troponins, myoglobin, galectin-3, micro ribonucleic acids and long non-coding ribonucleic acids are useful in the diagnosis, management and prognosis of heart failure patients. Besides them, imaging biomarkers derived from echocardiography, cardiac magnetic resonance, with late gadolinium enhancement, cardiac metaiodobenzylguanidine imaging using dual isotope (123)I and (99m)Tc, or CMR-derived T1 mapping can add relevant value to biomarker panels characterizing this devastating disease. Further studies are necessary to identify the most relevant imaging and serum biomarkers in a common panel that is easy to use, cost-effective and easily accessible for the patients.

\section{CONFLICT OF INTEREST}

Nothing to declare.

\section{ACKNOWLEDGEMENT}

This research was supported via the research grant no. P_34_465 financed by the Romanian Ministry of European Funds, the Romanian Government and the European Union.

\section{REFERENCES}

1. Maisel A, Mueller C, Nowak R, et al. Mid-region pro-hormone markers for diagnosis and prognosis in acute dyspnea: results from the $\mathrm{BACH}$
(Biomarkers in Acute Heart Failure) trial. J Am Coll Cardiol. 2010;55:20622076.

2. Januzzi JL Jr, Rehman SU, Mohammed AA, et al. Use of amino-terminal pro-B-type natriuretic peptide to guide outpatient therapy of patients with chronic left ventricular systolic dysfunction. J Am Coll Cardiol. 2011;58:1881-1889.

3. Ndumele CE, Matsushita K, Sang $\mathrm{Y}$, et al. N-Terminal Pro-Brain Natriuretic Peptide and Heart Failure Risk Among Individuals With and Without Obesity: The Atherosclerosis Risk in Communities (ARIC) Study. Circulation. 2016;133:631-638.

4. Wang YC, Yu CC, Chiu FC, et al. Soluble ST2 as a biomarker for detecting stable heart failure with a normal ejection fraction in hypertensive patients. J Card Fail. 2013:19:163-168.

5. Bayes-Genis A, de Antonio M, Vila J, et al. Head-to-head comparison of 2 myocardial fibrosis biomarkers for long-term heart failure risk stratification: ST2 versus galectin-3. J Am Coll Cardiol. 2014;63:158-166.

6. Gaggin HK, Szymonifka J, Bhardwaj A, et al. Head-to-head comparison of serial soluble ST2, growth differentiation factor-15, and highly-sensitive troponin $T$ measurements in patients with chronic heart failure. JACC Heart Fail. 2014;2:65-72.

7. Zhu ZD, Sun T. Association between growth differentiation factor-15 and chronic heart failure in coronary atherosclerosis patients. Genet Mol Res. 2015;14:2225-2233.

8. Mueller T, Leitner I, Egger M. Association of the biomarkers soluble ST2, galectin-3 and growth- differentiation factor-15 with heart failure and other non-cardiac diseases. Clin Chim Acta. 2015;445:155-160.

9. Chan MM, Santhanakrishnan R, Chong JP, et al. Growth differentiation factor 15 in heart failure with preserved vs. reduced ejection fraction. Eur J Heart Fail. 2016;18(1):81-88.

10. Scharnagl H, Kleber ME, Genser B, et al. Association of myeloperoxidase with total and cardiovascular mortality in individuals undergoing coronary angiography--the LURIC study. Int J Cardiol. 2014;174:96-105.

11. Gedikli O, Kiris A, Hosoglu Y, et al. Serum myeloperoxidase level is associated with heart-type fatty acid-binding protein but not troponin $\mathrm{T}$ in patients with chronic heart failure. Med Princ Pract. 2015;24:42-46.

12. Schiele F, Meneveau N, Seronde MF, et al. C-reactive protein improves risk prediction in patients with acute coronary syndromes. Eur Heart J. 2010;31:290-297.

13. Parrinello CM, Lutsey PL, Ballantyne CM, et al. Six-year change in highsensitivity C-reactive protein and risk of diabetes, cardiovascular disease, and mortality. Am Heart J. 2015;170:380-389.

14. Aulin J, Siegbahn A2, Hijazi Z, et al. Interleukin-6 and C-reactive protein and risk for death and cardiovascular events in patients with atrial fibrillation. Am Heart J. 2015;170:1151-1160.

15. Canada JM, Fronk DT, Cei LF, et al. Usefulness of C-Reactive Protein Plasma Levels to Predict Exercise Intolerance in Patients With Chronic Systolic Heart Failure. Am J Cardiol. 2016;117:116-120.

16. Kozdağ G, Ertaş G, Kiliç T, et al. Elevated level of high-sensitivity C-reactive protein is important in determining prognosis in chronic heart failure. Med Sci Monit. 2010;16:CR156-161.

17. Schmalgemeier $\mathrm{H}$, Bitter $\mathrm{T}$, Fischbach $\mathrm{T}$, et al. C-reactive protein is elevated in heart failure patients with central sleep apnea and CheyneStokes respiration. Respiration. 2014;87:113-120.

18. Ataoğlu HE, Yilmaz F, Uzunhasan I, et al. Procalcitonin: a novel cardiac marker with prognostic value in acute coronary syndrome. $J$ Int Med Res. 2010;38:52-61.

19. Kelly D, Khan SQ, Dhillon O, et al. Procalcitonin as a prognostic marker in patients with acute myocardial infarction. Biomarkers. 2010;15:325-331.

20. Sinning CR, Sinning JM, Schulz A, et al.; Association of serum procalcitonin with cardiovascular prognosis in coronary artery disease. Circ J. 2011:75:1184-1191.

21. Schiopu A, Hedblad B, Engström G, et al. Plasma procalcitonin and the risk of cardiovascular events and death: a prospective population-based study. J Intern Med. 2012;272:484-491.

22. Canbay A, Celebi OO, Celebi S, et al. Procalcitonin: a marker of heart failure. Acta Cardiol. 2015;70(4):473-478.

23. Loncar G, Tscholl V, Tahirovic E, et al. Should procalcitonin be measured routinely in acute decompensated heart failure. Biomark Med. 2015;9:651659.

24. Wang W, Zhang X, Ge N, et al. Procalcitonin testing for diagnosis and short-term prognosis in bacterial infection complicated by congestive heart failure: a multicenter analysis of 4,698 cases. Crit Care. 2014;18:R4.

25. Alba GA, Truong QA, Gaggin HK, et al. Diagnostic and Prognostic Utility of Procalcitonin in Patients Presenting to the Emergency Department with Dyspnea. Am J Med. 2016;129:96-104. 
26. Schuetz P, Kutz A, Grolimund E, et al. Excluding infection through procalcitonin testing improves outcomes of congestive heart failure patients presenting with acute respiratory symptoms: results from the randomized ProHOSP trial. Int J Cardiol. 2014;175:464-472.

27. Pascual-Figal DA, Manzano-Fernández S, Boronat M, et al. Soluble ST2 high-sensitivity troponin $\mathrm{T}$ - and $\mathrm{N}$-terminal pro-B-type natriuretic peptide: complementary role for risk stratification in acutely decompensated heart failure. Eur J Heart Fail. 2011;13:718-725.

28. Pascual-Figal DA, Casas T, Ordonez-Llanos J, et al. Highly sensitive troponin $\mathrm{T}$ for risk stratification of acutely destabilized heart failure. Am Heart J. 2012;163:1002-1010.

29. Grodin JL, Neale S, Wu Y, et al. Prognostic comparison of different sensitivity cardiac troponin assays in stable heart failure. Am J Med. 2015;128:276-282.

30. Haaf P, Reichlin T, Twerenbold R. Risk stratification in patients with acute chest pain using three high-sensitivity cardiac troponin assays. Eur Heart J. 2014;35:365-375

31. Hendgen-Cotta UB, Kelm M, Rassaf T. Myoglobin functions in the heart Free Radic Biol Med. 2014;73:252-259.

32. Wang Q, Liu F, Yang $X$, et al. Sensitive point-of-care monitoring of cardiac biomarker myoglobin using aptamer and ubiquitous personal glucose meter. Biosens Bioelectron. 2015;64:161-164.

33. Lee HY, Choi JS, Guruprasath P, et al. An Electrochemical Biosenso Based on a Myoglobin-specific Binding Peptide for Early Diagnosis of Acute Myocardial Infarction. Anal Sci. 2015;31:699-704.

34. Li X, Luo R, Jiang R, et al. The prognostic use of serum concentrations of cardiac troponin-I, CK-MB and myoglobin in patients with idiopathic dilated cardiomyopathy. Heart Lung. 2014;43:219-224.

35. Polat V, Bozcali E, Uygun T, et al. Diagnostic significance of serum galectin-3 levels in heart failure with preserved ejection fraction. Acto Cardiol. 2016:71:191-197.

36. Schindler El, Szymanski JJ, Hock KG, et al. Short- and Long-term Biologic Variability of Galectin-3 and Other Cardiac Biomarkers in Patients with Stable Heart Failure and Healthy Adults. Clin Chem. 2016;62:360-366.

37. Song X, Qian X, Shen M, et al. Protein kinase C promotes cardiac fibrosis and heart failure by modulating galectin-3 expression. Biochim Biophys Acta. 2015;1853:513-521.

38. $Y u X$, Sun $Y$, Zhao $Y$, et al. Prognostic value of plasma galectin-3 levels in patients with coronary heart disease and chronic heart failure. Int Heart $J$ 2015:56:314-318.
39. Lai KB, Sanderson JE, Izzat MB, et al. Micro-RNA and mRNA myocardial tissue expression in biopsy specimen from patients with heart failure. Int J Cardiol. 2015:199:79-83.

40. Devaux Y, Vausort M, McCann GP, et al. A Panel of 4 microRNAs Facilitates the Prediction of Left Ventricular Contractility after Acute Myocardia Infarction. PLoS One. 2013;8:e70644

41. Kumarswamy $R$, Bauters C Volkmann I, et al. Brief UltraRapid Communication (Clinical Track) Circulating Long Noncoding RNA, LIPCAR, Predicts Survival in Patients With Heart Failure. Circulation Research. 2014;114:1569-1575

42. Gu YL, Voors AA, Zijlstra F, et al. Comparison of the temporal release pattern of copeptin with conventional biomarkers in acute myocardial infarction. Clin Res Cardiol. 2011;100:1069-1076.

43. Meune C, Zuily S, Wahbi K, et al. Combination of copeptin and high-sensitivity cardiac troponin T assay in unstable angina and non-ST-segment elevation myocardial infarction: a pilot study. Arch Cardiovasc Dis. 2011:104:4-10.

44. Pozsonyi Z, Förhécz Z, Gombos T. Copeptin (C-terminal pro argininevasopressin) is an independent long-term prognostic marker in heart failure with reduced ejection fraction. Heart Lung Circ. 2015;24:359-367.

45. Vizzardi E, Bonadei I, Sciatti E, et al. Quantitative analysis of right ventricular (RV) function with echocardiography in chronic heart failure with no or mild RV dysfunction: comparison with cardiac magnetic resonance imaging. $J$ Ultrasound Med. 2015:34:247-255

46. Pellicori P, Zhang J, Lukaschuk E, et al. Left atrial function measured by cardiac magnetic resonance imaging in patients with heart failure: clinica associations and prognostic value. Eur Heart J. 2015;36:733-742.

47. Won E, Donnino R, Srichai MB, et al. Diagnostic Accuracy of Cardiac Magnetic Resonance Imaging in the Evaluation of Newly Diagnosed Heart Failure With Reduced Left Ventricular Ejection Fraction. Am J Cardiol. 2015;116:1082-1087

48. Bellevre D, Manrique A, Legallois D, et al. First determination of the heart-to-mediastinum ratio using cardiac dual isotope $\left({ }^{123} \mathrm{I}-\mathrm{MIBG} /{ }^{99} \mathrm{mTc}\right.$ tetrofosmin) CZT imaging in patients with heart failure: the ADRECARD study. Eur J Nucl Med Mol Imaging. 2015;42:1912-1919.

49. Narula J, Gerson M, Thomas GS, et al. ${ }^{123}$ I-MIBG Imaging for Prediction of Mortality and Potentially Fatal Events in Heart Failure: The ADMIRE-HFX Study. J Nucl Med. 2015;56:1011-1018

50. Rommel KP, von Roeder M, Latuscynski K et al. Extracellular Volume Fraction for Characterization of Patients With Heart Failure and Preserved Ejection Fraction. J Am Coll Cardiol. 2016;67:1815-1825. 